\title{
FORMAL METHODS IN PLC CONTROL DEMONSTRATED AT A FLEXIBLE MANUFACTURING LINE
}

\author{
Georg Frey \\ Institute of Automatic Control \\ University of Kaiserslautern, Germany \\ frey@eit.uni-kl.de
}

\begin{abstract}
This paper presents various formal approaches in the development of logic control algorithms. Programmable Logic Controllers (PLCs) are commonly used in automation and the algorithms running on them tend to be quite complex. This motivates the application of formal approaches to PLC programming. The approaches range from completely formalized design methods on the one end over the verification and validation (V\&V) of formally described controllers to V\&V of existing algorithms (developed in some industrially used PLC programming language) on the other end. This paper contains an overview and comparison of various design and verification approaches applied to a common example - the model of a flexible manufacturing line - presented at a session organized by the author at the American Control Conference 2002.
\end{abstract}

\section{INTRODUCTION}

Programmable Logic Controllers (PLCs) are the primary workhorse of industrial automation. Due to the growing complexity of control algorithms, and the growing power of formal approaches, there is a lot of interest in applying formal methods to PLC programming. In recent years, a lot of interdisciplinary work was aimed in this direction. This work results in the formalization of different steps in the control design process depending on what problems are to be solved (Frey 2000):

(1) The demand for reduced development times, and the possible reuse of existing software modules result in the need for a formal approach in the development of the PLC programs.

(2) The demand for high quality solutions and especially the application of PLC in safety-critical processes result in the need of validation procedures, i.e. formal methods to prove specific static and dynamic properties of the programs.

(3) The large numbers of already installed PLC programs together with the high expense of programming, leads to the search for verification and validation methods that can be applied directly to programs written in PLC specific programming languages like Ladder Diagram. 
This paper contains a summary and comparison of various design and verification approaches applied to a common example presented at a session organized by the author at the American Control Conference 2002. The idea is not to do a benchmark, which isn't even possible, due to the varying nature of the presented approaches but to give the reader an impression on how a reasonably simple logic control problem is viewed from quite different angles.

The paper is organized as follows: In the next Section the fischertechnik manufacturing line that serves as common example in all the presented approaches is introduced. Section 3 presents the various formal approaches in detail and Section 4 compares them. The paper is concluded by a summary and an outlook on further work in this area.

\section{FISCHERTECHNIK MANUFACTURING LINE}

The flexible manufacturing line used as example is a fischertechnik model consisting of three machines (drill, vertical mill with tool charger, horizontal mill) and three conveyors connecting the machines. There are 15 binary input signals and as many binary output signals. Several production scenarios for the line are possible. The model serves as Logic Control Testbed at the ERC/RMS at the University of Michigan (cf. Figure 1). A second model is installed at the University of Kaiserslautern, Germany (cf. Figure 2).

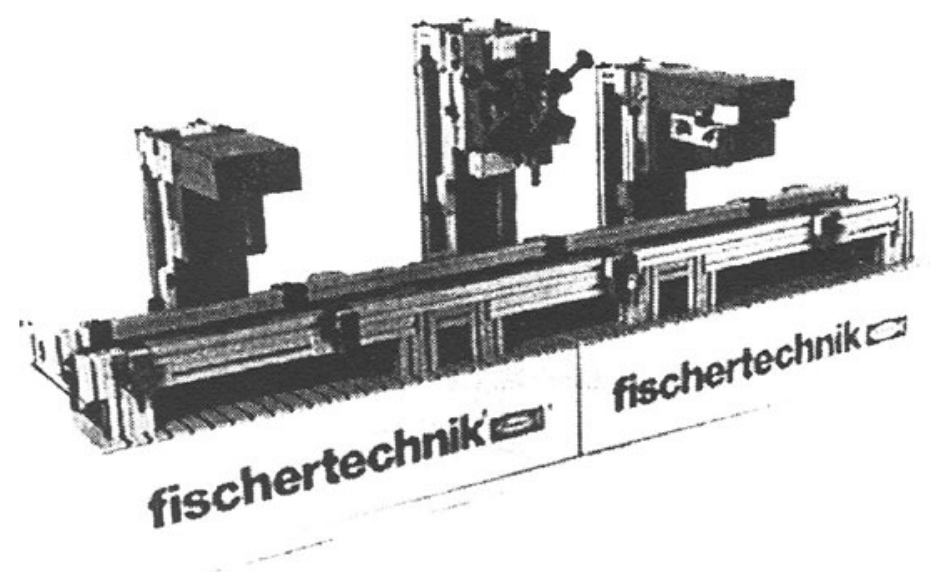

Figure 1 - FMS at the ERC in Michigan (Tilbury 2001)

Although the plants at the Universities of Michigan and Kaiserslautern are very similar there are a few reasons why neither the software nor the controllers are directly interchangeable. First of all, the Michigan line is controlled via a PC and programmed in $\mathrm{C}++$ language - generated from finite state machines, whereas the Kaiserslautern line is controlled either by a PLC programmed with Siemens STEP7 or an IPC with a proprietary real-time system that is programmable via standard IEC 61131-3 languages (John 2001) - generated from Petri nets (Frey 2000). Hence, the programs are not compatible. The controllers are not compatible with the other plant either because the plants use different voltage levels. 


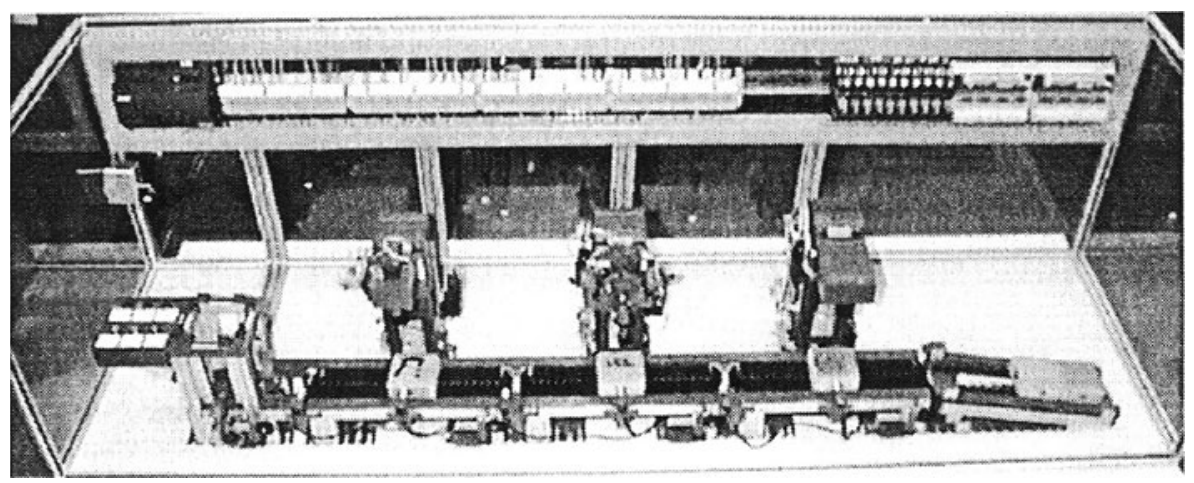

Figure 2 - FMS at the University of Kaiserslautern (Maas 2001)

The programs cannot be translated one-to-one into the language the other institute uses because the input and output tables are different as well. At the ERC in Michigan all the inputs are low active. On the Kaiserslautern plant either low active or high active inputs can be found. Below all the differences in hardware are listed sorted by the hardware units.

Table 1 - Differences in the Mechanical Setup of the Two Plants

\begin{tabular}{|c|c|c|}
\hline Units & FMS at Kaiserslautern & FMS at Ann Arbor \\
\hline User $\mathrm{V} / \mathrm{O}$ & Six push buttons with LEDs & One switch for on/off \\
\hline Feeder & $\begin{array}{l}\text { One motor to push the parts out } \\
\text { of the feeder; a reed sensor for } \\
\text { the position of the chain; one } \\
\text { infrared one way photoelectric } \\
\text { relay to see when the feeder is } \\
\text { empty; two reed sensors to } \\
\text { distinguish the parts }\end{array}$ & No feeder \\
\hline Conveyors & $\begin{array}{l}\text { Six inductive position sensors } \\
\text { in total; one at the entrance of } \\
\text { each conveyor and one in front } \\
\text { of each machine }\end{array}$ & $\begin{array}{l}\text { Four infrared sensors in } \\
\text { total, one at the entrance of } \\
\text { the first conveyor and one } \\
\text { in front of each machine }\end{array}$ \\
\hline $\begin{array}{l}\text { Machines } \\
\text { (Vertical } \\
\text { Mill) }\end{array}$ & $\begin{array}{l}\text { One limit switch at the tool } \\
\text { changer of the vertical mill to } \\
\text { recognize the working position } \\
\text { of one tool. }\end{array}$ & $\begin{array}{l}\text { An additional switch to } \\
\text { recognize when the changer } \\
\text { is rotating }\end{array}$ \\
\hline Storage & $\begin{array}{l}\text { At the entrance of the storage } \\
\text { for the finished parts is a two } \\
\text { way photoelectric relay that is } \\
\text { triggered when a part is directly } \\
\text { above the sensor }\end{array}$ & No storage \\
\hline Parts & $\begin{array}{l}\text { There are four different types } \\
\text { of workpieces }\end{array}$ & $\begin{array}{l}\text { No distinction between the } \\
\text { workpieces possible }\end{array}$ \\
\hline
\end{tabular}




\section{FORMAL APPROACHES}

\subsection{Design of a Controller for a FMS with SIPN and SFC, Validation and Verification of both Descriptions via Model-Checking}

In (Klein 2002) Stephane Klein, Xiying Weng, Georg Frey, Jean-Jaques Lesage, and Lothar Litz present the results of a joint project done at the ENS Cachan, France and the University of Kaiserslautern, Germany. The aim of this project is to assess the interest of performing formal validation on both the behavioral model and the implementation program of the same controller.

First, a formal model of the controller of the Flexible Manufacturing System is designed using Signal Interpreted Petri Nets (Frey 2002). This formal description is validated via model-checking (Klein 2001, Weng 2001). and then translated into a programming language of the IEC61131. Due to its graphical representation, Sequential Function Chart has been chosen. This program has been validated anew to make sure that the former validated behavior has not been modified during the translation phase. The second validation, again using model-checking with the same properties, shows that, even if the SFC is a simple translation of the former validated SIPN, errors can occur because of the different dynamics of the models.

This example shows that formal methods like validation are well suited for the design of control algorithms. But due to the different languages, and therefore the different semantics, used along the design process, a new step of validation has to be performed after each translation of the controller. None of these validation steps can be omitted if we want to be sure that What You Prove is What You Get.

\subsection{Verification of a Controller for a Flexible Manufacturing Line Written in Ladder Diagram}

In (De Smet 2002) Olivier de Smet and Olivier Rossi from the ENS in Cachan, France show how verification can work under real-life conditions. Rather than building a model of a controller and then analyzing it, they take a given controller written in Ladder Diagram as a starting point. The controller is supplied with the reference example in (Tilbury 2001).

In a first step the controller is translated in to a transition system. To do so, each primitive of the LD language and the LD execution rules are mathematically defined. Furthermore, a model of the PLCs execution behavior is build. The execution cycle of a PLC consists of the three steps: reading of the input image, processing of the algorithm, and writing of the output image (cf. Figure 3).

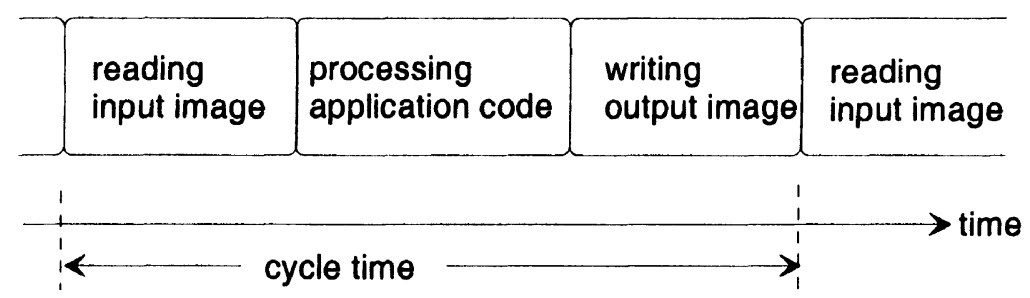

Figure 3 - Program Execution in a PLC 
With these definitions a parser was programmed that automatically generates the transition system from a given LD program. The resulting transition system is used as input for a model-checker.

To verify the system 90 properties are generated. The verification is done two times with different general approaches (Frey 2000). In the first run non-modelbased (i.e. without any model of the plant) and in the second run constrained-based (i.e. using very restricted knowledge about the plant as for example that the drill cannot be in the upper and lower end-position at the same time).

Using 4 constraints about the switches in the model all properties could be verified in less than a minute on a BDD of about 30000 nodes. In the non-modelbased approach the state space is several orders larger. Therefore the check of some properties needed several hours of computation time. Furthermore some properties were shown to be false. This is due to the fact, that "impossible behavior" of the plant is considered in the verification process. However it has to be pointed out that even physically impossible system states may be reported to the controller whenever there is a damaged sensor or a communication problem.

Therefore, De Smet and Rossi conclude that finding out whether a falsified property is caused by an error in the design or by abnormal system behavior is an open problem in model-checking approaches. Furthermore, they find modelchecking of LD programs to be effective for small to medium sized industrial systems but not feasible for large systems.

\subsection{Application of Specification Language SOL and Its Verification Method to A Flexible Manufacturing Line}

In (Ikkai 2002), Y. Ikkai, T. Nakashiba, and N. Komoda from Osaka University, Japan, show that conventional temporal logic descriptions of properties could be quite lengthy and error prone especially if exceptional processes in distributed control systems are described. An example for exceptional processes in the manufacturing line model is the unexpected entering of a new part. In this case the behavior of the line is different from the standard processing in the form that the unexpected part is moved to the end of the line without any machining operations. If the properties of the line are described in temporal logic formulae, most of them have to be rewritten to account for a special case like this. To avoid this problem, the Sequence Oriented Language (SOL), which is the intelligible language to describe specifications for distributed sequential control systems is proposed. SOL allows to describe the order of processes and exceptions from this order.

Verification using SOL is performed by a supporting tool that takes a model of the plant (described by finite state machines), the controller described by Function Blocks according to IEC61131 (translated into finite sate machines) and the SOL properties.

The presented tool not only allows verification but can also be used for the graphical design of the controller and the plant model. The application to the flexible manufacturing line shows the feasibility of the presented approach. 


\subsection{Reconfigurable Logic Control Using Modular FSMs: Design, Verification, Implementation, and Integrated Error Handling}

In (Shaw 2002) S. S. Shah form the University of California and E. W. Endsley, M. R. Lucas, and D. M. Tilbury from the University of Michigan describe the implementation and reconfiguration of controllers for the reference example.

They present an algorithm for the design of a modular controller that proceeds in six steps from the determination of the modular structure of the system under control to the implementation of the controller on the hardware.

The single modules of the logic control code are written using modular finite state machines and are verified to be internally correct before they are combined. After the combination of the resulting single finite state machine is verified before it is converted to $\mathrm{C}$ code for implementation.

The strength of this formalized modular development approach lies in the easy reconfiguration of the controller. Using the fischertechnik line it is shown how additional functionality can be integrated into the controller without writing it anew. This is a clear improvement to standard PLC programming languages where changes in the functionality of a controller are very hard to program and often a completely new controller is programmed instead.

\subsection{Quantitative and Qualitative Comparison of PLC for a Small Testbed with a Focus on Usability Issues}

In (Lucas 2002) M. R. Lucas and D. M. Tilbury from the University of Michigan describe similar programs written for the control of the fischertechnik manufacturing line in three languages: Ladder Diagram, Modular Finite State Machines, and Petri nets.

The aim of their work is to compare different programming languages or description formalisms from a users viewpoint. To do so they define some complexity metrics that are applicable to all the used languages. Furthermore the effort in writing and modifying the programs is measured. Finally the accessibility of the code, i.e. the ease of understanding the control logic, is compared.

There is no best formalism resulting from this multi-dimensional comparison. However the proposed framework for evaluating and comparing different formalisms can be used to find out whether new formalisms bring any advantages from a users point of view. Therefore it is a further step towards the integration of software quality concepts into logic controller development.

\section{COMPARISON OF THE PRESENTED APPROACHES}

\subsection{Verification and Validation}

In all of the presented approaches verification and validation of PLC programs is a main topic. Common to them is that they all use model-checking techniques. This clearly shows the importance of this V\&V method. The main difference lies in the 
formalism used to describe the control algorithm and the way in which the behavior of the plant is included.

For the control algorithms there are, on the one side, formal descriptions from academics as Petri Nets used in (Klein 2002) and Finite State Machines in (Shah 2002). On the other side, there are approaches that start from standard industrial descriptions as Ladder Diagram (De Smet 2002), Function Block Diagram (Ikkai 2002) or Sequential Function Chart (Klein 2002).

The behavior of the plant is either ignored (non-model-based $\mathrm{V} \& \mathrm{~V}$ ) as done in (Klein 2002), abstracted by some constraints (constrained-based V\&V) which showed to dramatically reduce the state-space during model-checking in (De Smet 2002) or completely described by a formal model (model-based V\&V) as presented in (Ikkai 2002).

A common problem in all approaches is the generation of formal properties to be verified on the system and the correct interpretation of the model-checking results. Furthermore it turns out that model-checking reaches its limits even for small controllers if there is no additional information on the plants behavior.

\subsection{Formal Design Approaches}

(Shaw 2002) propose a completely formalized design approach for reconfigurable controllers using modular finite state machines. Using this approach the reconfiguration of a controller is made much easier and faster as with conventional techniques.

In a comparative study (Lucas 2002) Ladder Diagrams, Petri Nets, and Modular Finite State Machines are used to develop a controller for the fischertechnik testbed. After the design the effort for writing the controller, the effort for changing the controller and the ease of understanding of the controller are evaluated. Further development of the presented metrics and the inclusion of even more formalisms could lead to a decision table that assists a user in finding the best language for the application at hand.

\section{CONCLUSIONS AND OUTLOOK}

In this paper several formal approaches to PLC programming are presented. All of them have been applied to the same control problem. The comparison of the approaches shows that verification and validation $(\mathrm{V} \& \mathrm{~V})$ of controllers is the main aim of formal methods in this area. However, new methods that try to improve the development process as a whole also begin to emerge. These development approaches try to avoid errors by formalized development procedures instead of finding them afterwards by V\&V.

Open problems to be addressed are the complexity of real-life controllers that are still out of range for the presented methods and the difficulties in finding the error if during V\&V properties turn out to be false.

To conclude, the application of more and more techniques from Software Engineering to PLC programming is the only way to cope with the increasing complexities in this application driven area. 
This survey should also be seen as a step to promoting the use of reference examples like the fischertechnik line in the presentation of new methods. A reference example allows the author and the reader to get to the relevant new points of the work rather fast, because the application is already known.

\section{ACKNOWLEDGEMENTS}

This paper would not have been written without Jim Christensen who had the idea of summarizing the results of the ACC session and invited me to present them at the BASYS conference. The paper would not have been possible without the valuable input from all the authors of the invited session on Formal methods in PLC control demonstrated at a flexible manufacturing line at the American Control Conference 2002.

\section{REFERENCES}

1. De Smet $\mathrm{O}$, Rossi O. Verification of a Controller for a flexible manufacturing line written in Ladder Diagram. Proceedings of the American Control Conference ACC2002, Anchorage, Alaska, pp.4147-4152, May 2002.

2. Frey G, Litz L. Formal methods in PLC programming. Proceedings of the IEEE Conference on Systems Man and Cybernetics SMC 2000, pp. 2431-2436, Nashville, Tennessee (USA), Oct. 8-11, 2000.

3. Frey, Georg. Automatic Implementation of Petri Net based Control Algorithms on PLC. Proceedings of the American Control Conference, ACC 2000, Chicago (IL), pp. 2819-3823, June 2000.

4. Frey, Georg. Design and formal Analysis of Petri Net based Logic Control Algorithms. Dissertation University of Kaiserslautern, Aachen: Shaker Verlag, 2002.

5. John KH, Tiegelkamp M. IEC 61131-3: Programming Industrial Automation Systems. Berlin, New York: Springer, 2001.

6. Klein S, Weng X, Frey G, Lesage JJ, Litz L. Design of a controller for a FMS with SIPN and SFC. Validation and verification of both descriptions via model-checking. Proceedings of the American Control Conference ACC2002, Anchorage, Alaska, pp.4141-4146, May 2002.

7. Klein, Stéphane. A case study in design and formal verification of control algorithms using interpreted Petri Nets and SFC. D.E.A. de Production Automatisée, Ecole Normale Supérieure Cachan, June 2001.

8. Lucas MR, Tilbury DM. Quantitative and Qualitative Comparison of PLC programs for a small testbed with a focus on usability issues. Proceedings of the American Control Conference ACC2002, Anchorage, Alaska, pp.4165-4171, May 2002.

9. Maas H, Frey G. Documentation and Control Scenarios for a Flexible Manufacturing Line. Technical Report 117/2001, Institute of Automatic Control, University of Kaiserslautern, Dec 2001.

10. Shah SS, Endsley EW, Lucas MR, Tilbury DM. Reconfigurable Logic Control using Modular FSMs: Design, Verification, Implementation, and Integrated Error Handling. Proceedings of the American Control Conference ACC2002, Anchorage, Alaska, pp.4153-4158, May 2002.

11. Tilbury, Dawn. Logic Control Testbed 2001 http://www-personal.engin.umich.edu/ tilbury/testbed/

12. Weng $X$, Litz L. Model checking of Signal Interpreted Petri Nets. Proceedings of the IEEE international Conference on Systems Man and Cybernetics, SMC 2001, Tucson (AZ), USA, pp. 2748-2752, Oct. 2001.

13. Y. Ikkai Y, Nakashiba T, Komoda N. Application of Specification Language SOL and its Verification Method to a Flexible Manufacturing Line. Proceedings of the American Control Conference ACC2002, Anchorage, Alaska, pp.4159-4164, May 2002. 